\title{
Semirigid ureteroscopy prior retrograde intrarenal surgery (RIRS) helps to select the right ureteral access sheath
}

\author{
Ioannis Boulalas, Mauro De Dominicis, Lorenzo Defidio \\ Department of Urology, Cristo Re Hospital, Rome; Italy.
}

\begin{abstract}
Summary Objective: To evaluate ureteral compliance through semirigid ureteroscopy (sURS) in order to select the proper ureteral access sheath (UAS) size for retrograde intrarenal surgery (RIRS).

Patients and methods: In a prospective study, 100 consecutive patients selected for elective sURS or RIRS were recruited. Each patient, initially underwent $9.5 \mathrm{Fr}$ sURS with a safety guidewire $3 \mathrm{Fr}$, in order to estimate ureteral compliance. If the ureter was compliant, a gently passage of a 12/14Fr UAS was attempted. If the ureter was not deemed compliant, passage of either a smaller UAS or a smaller semirigid 7Fr or a flexible $7.5 \mathrm{Fr}$ or a digital $8.5 \mathrm{Fr}$ scope with and without safety guidewire, was attempted. Age, gender, disease location, prestenting, previous RIRS and/or stone elimination, hydronephrosis, ureteral strictures, unsuccessful procedures, and complications, were analyzed as possible correlated factors of ureteral compliance.

Results: In 77 patients the ureter was deemed compliant $\geq 14 \mathrm{Fr}$. Of the preoperative factors that were examined, stent placement before RIRS $(P<0.002)$, previous RIRS $(P=0.000)$ and previous stone elimination $(P=0.004)$, correlated with ureter $\geq 14 \mathrm{Fr}$. Ureteral lithiasis $(P<0.001)$, ureteral strictures $(P<0.05)$, unsuccessful procedures $(P<0.005)$ and complications $(P=0.01)$ correlated with ureter $<14 \mathrm{Fr}$. The complication rate was $10 \%$ (10 patients) with ureteral injuries grade I in 9 patients and grade III in 1 patient according to the endoscopic grading system. Age, gender, hydronephrosis and urothelial carcinoma (UC) had no influence.

Conclusions: sURS performed before RIRS allows selection of the right ureteral access sheath (UAS) and avoidance of major complications. Pre-stenting, previous RIRS and stone elimination history are all factors correlating with a compliant ureter.
\end{abstract}

KEY WORDS: Semirigid ureteroscopy; Retrograde intrarenal surgery (RIRS); Ureteral compliance; Ureteral access sheath (UAS).

Submitted 8 November 2017; 19 November 2017

\section{INTRODUCTION}

The management of intra-renal collecting system pathology has changed radically over the recent decades mainly due to increasing use of flexible uretero-renoscopy (fURS), constituting one of the most dynamic fields of endourology. Thanks to technologic improvements in the endoscopic armamentarium, flexible uretero-renoscopic approaches to the kidney have evolved from a mere diagnostic tool, to a complex diagnostic and therapeutic procedure in the entire upper tract collecting system (1).
Nowadays, retrograde intrarenal surgery (RIRS) $(2,3)$ is used in the treatment of urinary lithiasis (4), upper tract urinary tumors $(5,6)$, as well as in special circumstances such as pregnancy, anatomic malformations, coagulopathy or solitary kidney $(7,8)$.

Urinary stones disease poses a significant health care burden in a working-age population. A recent analysis of National Health and Nutrition Examination Survey (NHANES) data in the United States from 2007 to 2010 reported that the prevalence had increased to $8.8 \%$ (10.6\% among men vs $7.1 \%$ among women), compared with 5.5\% in NHANES III (1988-1994) (9).

Upper tract urothelial carcinoma (UTUC) constitutes approximately 5-6\% of all urothelial malignancies. Ureteral tumors represent approximately $25 \%$ of UTUCs (10).

In 2013, the European Association of Urology (EAU) for the first time included RIRS as a viable treatment option for renal stones, even larger than $2 \mathrm{~cm}$ in diameter (11). Moreover, the UTUC EAU Guidelines support renal-sparing surgery in imperative cases and low-risk patients (12). The ureteral access sheath (UAS) was introduced as a means of passing a flexible uretero-renoscope into the distal ureter. Its use also facilitates multiple re-entries into the kidney, improves fluid outflow, thereby reducing the intrarenal pressure, decreases operative time, increases stone clearance, and protects the endoscope from damage $(13,14)$. However the routine use of a UAS is matter of debate (11).

Limited data is available to predict which patients selected for RIRS may have a difficult ureter and in which cases success is most probable.

The hypothesis that endoscopic evaluation of ureteral size may help urologists to select the proper UAS size for RIRS, was prospectively tested and possible correlating factors for success or failure were analyzed.

\section{Patients and methods}

This study was carried out at 'Cristo Re' Hospital in Rome, after institutional review board approval was obtained, by two experienced urologists, who treated a similar number of patients. Both surgeons were present during the procedures, thus avoiding any difference in the assessment accuracy. A total of 100 consecutive patients (72 males and 28 females) between March 2016 and September 2016, with urinary lithiasis, upper urinary tract tumors, hematuria and ureteral stenosis planned for elective semi- 
rigid ureterorenoscopy or RIRS were included in the study. All patients had a clinical evaluation, urine dipstix analysis with additional culture and sensitivity if a urinary tract infection (UTI) was suspected, a measurement of serum creatinine level, abdominal ultrasonography (US) and a plain abdominal X- ray. Additional computed tomography (CT) was used, according to the level of serum creatinine and stone radiolucency. Patients were placed in the lithotomy position and received prophylactic parenteral antibiotics before the procedure, which was performed under spinal or general anesthesia. The standard technique was initiated with rigid cystoscopy, followed by ureteral catheterization of the renal unit in question with an end-hole 5Fr catheter (Pollack Cook Urological, Spencer, $I N)$. A retrograde pyelogram was performed to define the anatomy and visualize any filling defect. A hydrophilic tipped guidewire 0.035/150 cm (Sensor ${ }^{\circledR}$, Boston Scientific, Marlborough, MA, USA) was passed via the ureteral catheter, just to the renal pelvis under fluoroscopic guidance, and set aside as a safety wire, and then the cystoscope and the retrograde catheter were removed. A second hydrophilic tipped guidewire was introduced into the ureteral orifice through the 9.5Fr Storz semirigid ureteroscope's (27002L Karl Storz, Rietheim-Weilheim, Germany) working channel that allowed retrograde ureteral access with relative ease. A gentle attempt was made for ureteroscopic access between these two guidewires. This maneuver allowed the optical ureteral dilation, permitting an easier upper tract access as well as inspection for the presence of pathology such as stones, strictures, or tumors, offering the possibility of treatment along all the ureter and, often, also in the kidney. At the same time, ureteral compliance and caliber were assessed in order to select the proper UAS size, avoiding ureteral injuries. The ureteroscope was withdraw and the UAS 12\14 Fr $35 \mathrm{~cm}$ (Flexor, Cook Urological, Spencer, IN) was introduced gently into the ureter under fluoroscopic control, by gliding over the working guidewire. In patients with ureteral stent, a PTFE guidewire 0.035/145 cm (Cook Urological, Spencer, IN) was inserted and advanced to the kidney through the distal end of the stent which had been brought to the urethral meatus. Finally the inner UAS obturator with the guidewire was removed and the 7Frl $43 \mathrm{~cm}$ Storz semirigid, or flexible or digital ureteroscope (27000L, Flex- $X^{2}$ or, Flex- $X^{C}$ Karl Storz, Rietheim-Weilheim, Germany) was inserted to complete the operation with a Holmium or Thulium Iaser device (15). We selected $12 \backslash 14 \mathrm{Fr}$ as the best UAS size because it can accept all the flexible and some semirigid ureteroscopes, while maintaining a good drainage in order to keep low intrarenal pressures. In difficult cases, when the ureter didn't accept the larger UAS, first we tried to remove the safety guide wire with the purpose to get more room. If still the passage of the larger UAS was not easy, we used a smaller UAS (11 $13 \mathrm{Fr}$ Flexor, Cook Urological, Spencer, IN). If this attempt was also without success, we then tried to introduce the flexible or digital scope, with or without the safety guidewire, under visual or fluoroscopic control. If again this attempt was encountered with resistance, we stopped the procedure, put a DJ stent and tried the week after.

If the patient was not planned for RIRS, but presented with an impacted ureteral stone with hydronephrosis, we

still inserted an access sheath just below the stone and we performed a lasertripsy of the stone with a semirigid scope $9.5 \mathrm{Fr}$ inside the access sheath with a safety guidewire 3Fr aside, in order to have a continuous flow with gravity irrigation and still low retro-pulsion pressures and expulsion of fragments retrieval through the sheath.

The primary outcome was the assessment of the ureteral compliance to identify a potential difficult ureter. A compliant ureter was defined as a ureter $\geq 14 \mathrm{Fr}$ if accepted easily the passage of a semirigid scope $9.5 \mathrm{Fr}$ with a safety guidewire 3Fr aside.

Patient's data collected, included age, gender, side, hydronephrosis, stone location (renal, ureteral, renal \& ureteral), UTUC, presence of ureteral strictures, an indwelling ureteral stent, previous RIRS, and previous stone elimination history.

\section{Statistical analyses}

The significance of possible factors affecting ureteral compliance was analyzed. Statistical analysis was performed with SPSS 20 adopting the chi-square test for nominal variables and the Mann-Whitney U test for continuous variables. Statistical significance was considered at $\mathrm{p}<0.05$.

\section{RESULTS}

The study group included 100 consecutive patients that were treated at 'Cristo Re' Hospital in Rome. Patient's data are presented in Table 1.

$82 \%$ and $18 \%$ of the patients were submitted to RIRS and sURS respectively, with or without use of UAS. Hydronephrosis was present in 56\% of the patients while in $29 \%$ a DJ stent was inserted before (24 patients) and during surgery ( 5 patients) due to acute obstruction, insistent pain, fever, pus, ureteral injury, ureteral stricture and non compliant ureter.

A compliant ureter with a diameter $\geq 14 \mathrm{Fr}$ was present in 77 patients because a 12/14Fr UAS was inserted gently with or without a safety guidewire, either a 9.5Fr semirigid ureteroscope with a safety guidewire was used (objective evaluation). In 23 patients, the ureter was non

\section{Table 1.}

Patient demographics.

\begin{tabular}{|lc|}
\hline Patient, $\mathbf{n}$ & $\mathbf{1 0 0}$ \\
\hline Mean age years (range) & $54(16-89)$ \\
\hline Men & 72 \\
\hline Women & 28 \\
\hline Ureteral stone & 18 \\
\hline Renal stone & 45 \\
\hline Ureteral \& Renal Stone & 12 \\
\hline UTUC, hematuria & 22 \\
\hline Ureteral stricture & 23 \\
\hline Side right/left & $51 / 49$ \\
\hline Hydronephrosis (\%) & 56 \\
\hline Indwelling Double-J stent, $n(\%)$ & 29 \\
\hline Previous RIRS, $n(\%)$ & 29 \\
\hline Previous stone elimination, $n(\%)$ & 42 \\
\hline
\end{tabular}


Table 2.

Results of ureteral calibration.

\begin{tabular}{|c|c|c|}
\hline & $\begin{array}{l}\text { Patients } \\
77\end{array}$ & $\begin{array}{l}\text { Ureter diameter } \\
\quad \geq 14 \mathrm{fr}\end{array}$ \\
\hline $\begin{array}{l}\text { Gently passage of a 12/14Fr UAS with or } \\
\text { without a safety guidewire }\end{array}$ & 73 & $\geq 14 \mathrm{fr}$ \\
\hline \multirow[t]{2}{*}{$\begin{array}{l}\text { Gently passage of a } 9.5 \text { Semirigid Ureteroscope } \\
\text { with a safety guidewire }\end{array}$} & 4 & $\geq 14 \mathrm{fr}$ \\
\hline & $\begin{array}{l}\text { Patients } \\
23\end{array}$ & $\begin{array}{l}\text { Ureter diameter } \\
\quad<14 \text { fr }\end{array}$ \\
\hline $\begin{array}{l}\text { Gently passage of a 11/13F UAS after } \\
\text { removal of the safety guidewire }\end{array}$ & 1 & $<14 \mathrm{fr}$ \\
\hline $\begin{array}{l}\text { Gently passage of a 7Fr Semirigid, flexible or } \\
\text { or digital Uretero-renoscope with or without } \\
\text { a safety guidewire }\end{array}$ & 22 & $<14 \mathrm{fr}$ \\
\hline
\end{tabular}

Table 3.

Complications.

\begin{tabular}{|lcc|}
\hline & Patient $\mathbf{1 0}$ & Patients, grade \\
\hline Guidewire & 8 & 8 Grade I \\
\hline UAS & 2 & 1 Grade I \\
\hline & & 1 Grade III \\
\hline
\end{tabular}

compliant with a diameter $<14 \mathrm{Fr}$ due to insertion of either a 11/13Fr UAS after removal of the safety guidewire, or of a 7Fr semirigid or flexible or digital ureteroscope with or without safety guidewire (Table 2). In 7 patients, we had to stop the procedure and a DJ stent or nephrostomy tube was introduced. Complications were noted in 10 patients. Guidewire-induced ureteral damage occurred in 8 patients with grade I lesions, while UAS- induced ureteral injuries occurred in 2 patients with grade I lesion in one case and grade III in the other according to the endoscopic grading system (16) (Table 3).

Different factors that may influence the ureteral compliance were examined. The following parameters correlated statistically with a ureter $\leq 14 \mathrm{~F}$ : ureteral lithiasis, ureteral stricture, unsuccessful procedures and complications (Table 4)

Table 4.

Parameters of compliant ureter.

\begin{tabular}{|lccccc|}
\hline & $\begin{array}{c}\text { Compliant } \mathbf{1 4 F r} \\
\mathbf{7 7} \text { pts }\end{array}$ & $\begin{array}{c}\text { Non compliant < 14Fr } \\
\text { 23 pts }\end{array}$ & p-value \\
\hline Age & 57.6 & & 52.1 & 0.1 \\
\hline Sex (males) & 53 & $(69 \%)$ & 19 & $(83 \%)$ & 0.4 \\
\hline Ureteral lithiasis & 13 & $(17 \%)$ & 14 & $(61 \%)$ & $<0.001$ \\
\hline Pre-stenting & 28 & $(36 \%)$ & 1 & $(4 \%)$ & $<0.002$ \\
\hline Hydronephrosis & 39 & $(51 \%)$ & 17 & $(74 \%)$ & 0.1 \\
\hline First time RIRS & 47 & $(61 \%)$ & 15 & $(65 \&)$ & 1 \\
\hline Previous RIRS & 26 & $(34 \%)$ & 3 & $(13 \%)$ & 0.000 \\
\hline Number of patients with & 22 & $(28 \%)$ & 4 & $(17 \%)$ & 0.6 \\
renal stones $\leq 1.5$ cm (\%) & & & & & 1 \\
\hline Number of patients with & 2 & $(2.6 \%)$ & 2 & $(8.6 \%)$ & \\
\hline ureteral stones $\leq 1 \mathrm{~cm}(\%)$ & 18 & $(23 \%)$ & 4 & $(17 \%)$ & 0.5 \\
\hline UTUC & 1 & $(1.3 \%)$ & 22 & $(95 \%)$ & $<0.05$ \\
\hline Ureteral stricture & 29 & $(38 \%)$ & 11 & $(49 \%)$ & 0.004 \\
\hline Previous stone elimination & 0 & & 7 & $(30 \%)$ & $<0.005$ \\
\hline Unsuccessful procedures & 5 & $(6.5 \%)$ & 5 & $(21 \%)$ & $<0.01$ \\
\hline Complications & & & & & \\
\hline
\end{tabular}

\section{Discussion}

Over the last decade the advancements in technology and digital optics have led to an increasing role of fURS in the treatment of upper urinary tract pathology. A ureteral access sheath is an important tool during RIRS, because it lowers intra-renal pressure, facilitates ureteral re-entry, decreases costs, reduces operative time, and improves flexible uretero-renoscope longevity (17).

There are different UAS's in the market with different characteristics, sizes and lengths. Factors that are important in clinical application include a lubricated outer coating to facilitate entry, a lower friction inner coating for easy uretero-renoscope insertion, and a reinforced wall to decrease sheath kinking and bulking (18).

The standard UAS has an external diameter of 14Fr, which is larger than the median 9Fr to 10Fr diameter of non stented ureters, as evaluated on imaging studies (19). Insertion of a UAS depends on the ureter status and on its anatomic variants. Failure rates of primary access due to difficult impassable ureter range from 8\%-10\% $(20,21)$.

Lallas et al. in animal models showed that the over distention created by the UAS caused a transient decrease in ureteral blood flow which restored at a basal level by the compensatory mechanisms of the ureteral wall and the integrity of the ureter was preserved. However, care must be taken for selecting an appropriate-size sheath and the duration of surgery should not be long because the risk of stricture development has not been clearly put forward (22).

Viers et al. examined the association between clinic-radiographic features and need for pre-stenting due to inability of the ureter to accommodate the ureteroscope or the UAS, and found 17\% incidence of primary upper tract access failure. Prior ipsilateral ureteral surgery and stenting were protective whereas $<50 \%$ ureteral opacification was associated with an increased risk of access failure (23). Some authors position the UAS under fluoroscopic guidance with the application of reasonable strength on the working guidewire without the performance of semi-rigid ureteroscopy (23) whereas others calibrate the ureter with an $8 \mathrm{~F} / 10 \mathrm{~F}$ coaxial dilator (Boston Scientific) (24). Additional strategies, that are used to go into the ureter, include routine stent placement before RIRS which entails two-stage procedure, sequential ureteral dilators or balloon dilation with significant risk of ureteral injury (25).

In a prospective study in 248 patients undergoing sURS and fURS Mogilevkin et al. found that in $22 \%$ of patients the UAS was not easily passed. Factors of successful insertion were: older age, presenting and previous same-side ureteroscopy (25).

Traxer and Thomas, in a two center prospective review, collected data on 359 patients who received a 12/14Fr UAS before a RIRS for renal stones. The authors identified and classified any ureteral injuries. UAS-induced ureteral lesions occurred in $46.5 \%$ of patients, with complete wall perforation in $13.4 \%$. 
The authors conclude that is imperative to visualize the ureter at the end of the procedure and that pre-stenting can reduce the risk of severe injury to the ureter. Risk factors for high grade lesions included age, male gender, and lack of preoperative stent (26).

However, in another work on 2239 patients treated with fURS from the Clinical Research Office of the Endourological Society (CROES), Traxer et al. reported that UAS usage did not increase the risk of ureteral wall damage (27).

Guzelburc et al. in a prospective study investigated ureteral injuries by placing two different UAS (9.5/11.5Fr-12/14Fr) during retrograde intrarenal surgery (RIRS) for renal stones. The researchers identified $41,6 \%$ ureteral lesions, with deep tear of the mucosal and submucosal layer in $2.97 \%$ (PULS grade 2) of patients and no injuries of grade 3+. PULS grade 2 patients were all males and a DJ stent was placed preoperatively in all cases $(28,29)$.

Yet, there have been few studies demonstrating significant long-term ureteral damage following the use of a UAS. In the past, one ureteropelvic junction stricture (1.4\%) was noted to occur during a mean follow-up of 11 months after UAS use (30).

In our study $77 \%$ of the patients had a compliant ureter $\geq 14 \mathrm{Fr}$, and 23 a not compliant ureter $<14 \mathrm{Fr}$.

Age, sex, stone size, hydronephrosis, or UTUC had no influence on ureteral compliance in this analysis.

Patients with an indwelling DJ stent, previous RIRS history and previous stone elimination accommodated easily a $12 / 14$ Fr UAS.

The presence of ureteral stone adversely affected compliance due to acute or chronic inflammation.

The ureteral stricture caused by impacted stones, recurrences, or conservative treatment of UTUC's were also consistent with a non compliant ureter $<14 \mathrm{Fr}$.

Finally the unsuccessful procedures and the complications were correlated with a not compliant ureter.

The overall low incidence of unsuccessful procedures (7\%) is due to the adoption of some tricks before interrupting the procedure, such as the removal of safety guidewire or the use of a smaller UAS or ureteroscope.

Only in 8 patients complications were caused by the guidewire and only in 2 by the UAS, of which only one had a grade 3 lesion.

The low complications rate (10\%), most of them of grade $\mathrm{I}$, is probably due to the respect of the two fundamental endourological rules: the first one is to adapt the instrumentldevice to the ureter and not the ureter to the instrument, and the second one is to never force in the introduction or extraction of instrument or device.

In the current study the importance of our technique to perform sURS prior to UAS insertion during RIRS is demonstrated, as it permits ureteral inspection and allows assessment of ureteral compliance.

Those with a low likelihood for effective 12/14Fr UAS include patients with ureteral stricture and ureteral stone. However, this study presents some limitations due to the small number of patients. Probably the data can be different in a larger cohort of patients. Also, we didn't have a follow-up of patients to report possible postoperative complications.

\section{Conclusions}

Semirigid ureteroscopy performed prior retrograde intrarenal surgery (RIRS) is an outstanding tool for evaluation of ureteral compliance, allowing selection of the correct UAS size. A compliant ureter $\geq 14 \mathrm{Fr}$ was found in 3/4 of the patients. Parameters of successful insertion of a 12/14Fr UAS were an indwelling DJ stent, and a history of previous RIRS or stone elimination while the presence of a ureteral stone or stricture, unsuccessful procedures and complications significantly predicted a non compliant ureter $<14 \mathrm{Fr}$.

\section{REFERENCES}

1. Oberlin DT, Flum AS, Bachrach L, et al. Contemporary surgical trends in the management of upper tract calculi. J Urol. 2015; 193:880.

2. Patel A, Fuchs GJ. Expanding the horizons of SWL through adjunctive use of retrograde intrarenal surgery: New techniques and indications. J Endourol. 1997; 11:33.

3. Shin R, Lipkin M, Preminger G. Disposable devices for RIRS: Where do we stand in 2013? What do we need in the future? World J Urol. 2015; 33:241

4. Ordon M, Urbach D, Mamdani $M$, et al. The surgical management of kidney stone disease: a population based time series analysis. J Urol. 2014; 192:1450.

5. Cornu JN, Rouprêt M, Carpentier X, et al. Oncological control obtained after exclusive flexible ureteroscopic management of upper urinary tract urothelial carcinoma. World J Urol. 2010; 28:151.

6. Cutress ML, Stewart GD, Wells-Cole S, et al. Long-term endoscopic management of upper tract uothelial carcinoma (UTUC): 20years single-centre experience. BJU Int. 2012; 110:1608.

7. Giusti G, Proietti S, Cindolo L, et al. Sky is not the limit for ureteroscopy: extending the indications and special circumstances World J Urol. 2015; 33:257.

8. Giusti G, Proietti S, Cindolo L, et al. Is retrograde intrarenal surgery a viable treatment option for renal stones in patients with solitary kidney? World J Urol. 2015; 33:309.

9. Shoag J, Tasian GE, Goldfarb DS, et al. The new epidemiology of nephrolithiasis. Adv Chronic Kid Dis. 2015; 22:273.

10. Bader MJ, Sroka R, Gratzke C, et al. Laser therapy for upper urinary tract transitional cell carcinoma: indications and management. Eur Urol. 2009; 56:65.

11. Türk C, Petrik A, Sarica K, et al. EAU Guidelines on Interventional Treatment for Urolithiasis. Eur Urol. 2016; 69:475.

12. Rouprêt M, Babjuk M, Compérat E, et al. European Association of Urology Guidelines on Upper Urinary Tract Urothelial Cell Carcinoma. Update Eur Urol. 2015; 68:868.

13. Stern JM, Yiee J, Park S. Safety and efficacy of ureteral access sheaths. J Endourol. 2007; 21:119.

14. Bach C, Nesar S, Kumar P, et al. The new digital flexible ureteroscopes: 'size does matter'- increased ureteric access sheath use. Urol Int. 2012; 89:408.

15. Defidio L, De Dominicis M, Gianfrancesco D, et al. First collaborative experience with thulium Laser ablation of localized upper urinary tract urothelial tumors using retrograde intra-renal surgery Arch Ital Urol Androl. 2011; 83:147.

16. Karakan T, Kilinc MF, Demirbas A, et al. Evaluating Ureteral 
Wall Injuries with Endoscopic Grading System and Analysis of the Predisposing Factors. J Endourol. 2016; 30:375.

17. Defidio L, De Dominicis M, Di Gianfrancesco L, et al. Improving Flexible Ureterorenoscope Durability Up to 100 Procedures. J Endourol. 2012; 26:1329.

18. Khanna $R$ and Monga M. Instrumentation in endourology. Ther Adv Urol. 2011; 3:119.

19. Zelenko N, Coll D, Rosenfeld AT, et al. Normal ureter size on unenhanced helical. CT AJR. 2004; 182:1039.

20. Cetti RJ, Biers S, Kcoghane SR. The difficult ureter: what is the incidence of presenting? Ann R Coll Surg Engl. 2011; 93:31.

21. Bourdoumis A, Tanabalan C, Goyal A, et al. Stent and come back or balloon dilate and proceed with ureteroscopy? What does the evidence say? Urology. 2014; 83:1.

22. Lallas C, Auge B, Raj G, et al. Laser Doppler flowmetric determination of ureteral blood flow after ureteral access sheath placement J Endourol. 2002; 16:583.

23. Viers B, Viers L, Hull N, et al. The difficult ureter: Clinical and radiographic characteristics associated with upper urinary tract access at the time of ureteroscopic stone treatment. Urology. 2015; 86:878.

24. Goldberg H, Holland R, Tal R, et al. The impact of retrograde intrarenal surgery for asymptomatic renal stones in patients undergoing ureteroscopy for a symptomatic ureteral stone J Endourol 2013; $27: 970$

25. Mogilevkin Y, Sofer M, Margel D, et al. Predicting an effective ureteral access sheath insertion: A bicenter prospective study. J Endourol. 2014; 28:1414.

26. Traxer O. and Thomas A.Prospective evaluation and classification of Ureteral wall injuries resulting from insertion of a ureteral access sheath during retrograde intrarenal surgery. J Urol. 2013; 189:580.

27. Traxer O, Wendt-Nordahl $G$, Sodha $H$, et al. Differences in renal stone treatment and outcomes for patients treated either with or without the support of a ureteral access sheath: The Clinical Research Office of the Endourological Society Ureteroscopy Global Study. World J Urol. 2015; 33:2137.

28. Guzelburc V, Guven S, Boz MY, et al.Intraoperative Evaluation of Ureteral Access Sheath-Related Injuries Using Post-Ureteroscopic Lesion Scale J Laparoendosc Adv Surg Tech A. 2016; 26:23.

29. Schoenthaler M, Buchholz N, Farin E, et al. The PostUreteroscopic Lesion Scale (PULS): a multicenter video-based evaluation of inter-rater reliability. World J Urol. 2014; 32:1033.

30. Delvecchio FC, Auge BK, Brizuela RM, et al. Assessment of stricture formation with the ureteral access sheath. Urology. 2003; 61:518.

\section{Correspondence}

Boulalas Ioannis, MD, PhD (Corresponding Author)

iboulalas@yahoo.gr

De Dominicis Mauro, MD

dedominicism@alice.it

Defidio Lorenzo, MD

defidio@tin.it

Department of Urology - Cristo Re Hospital

Via delle Calasanziane 25, 00167 Rome, Italy 